\title{
BMJ Open Does physical exposure throughout working life influence chair-rise performance in midlife? A retrospective cohort study of associations between work and physical function in Denmark
}

\author{
Anne Møller, ${ }^{1,2,3}$ Susanne Reventlow, ${ }^{3}$ Åse Marie Hansen, ${ }^{2,4}$ Lars L Andersen, ${ }^{2}$ \\ Volkert Siersma, ${ }^{3}$ Rikke Lund, ${ }^{4,5}$ Kirsten Avlund, ${ }^{4,5, \dagger}$ Johan Hviid Andersen, ${ }^{6}$ \\ Ole Steen Mortensen ${ }^{1,2}$
}

To cite: Møller A, Reventlow S, Hansen ÅM, et al. Does physical exposure throughout working life influence chair-rise performance in midlife? A retrospective cohort study of associations between work and physical function in Denmark. BMJ Open 2015;5: e009873. doi:10.1136/ bmjopen-2015-009873

- Prepublication history and additional material is available. To view please visit the journal (http://dx.doi.org/ 10.1136/bmjopen-2015009873).

†KA passed away in September 2013, former affiliation.

Received 3 September 2015 Revised 18 September 2015 Accepted 21 September 2015

CrossMark

For numbered affiliations see end of article.

Correspondence to Dr Anne Møller; annemoller1972@gmail.com

\section{ABSTRACT}

Objectives: Our aim was to study associations between physical exposures throughout working life and physical function measured as chair-rise performance in midlife.

Methods: The Copenhagen Aging and Midlife Biobank (CAMB) provided data about employment and measures of physical function. Individual job histories were assigned exposures from a job exposure matrix. Exposures were standardised to ton-years (lifting $1000 \mathrm{~kg}$ each day in 1 year), stand-years (standing/ walking for $6 \mathrm{~h}$ each day in 1 year) and kneel-years (kneeling for $1 \mathrm{~h}$ each day in 1 year). The associations between exposure-years and chair-rise performance (number of chair-rises in $30 \mathrm{~s}$ ) were analysed in multivariate linear and non-linear regression models adjusted for covariates.

Results: Mean age among the 5095 participants was 59 years in both genders, and, on average, men achieved 21.58 ( $\mathrm{SD}=5.60)$ and women 20.38

$(S D=5.33)$ chair-rises in $30 \mathrm{~s}$. Physical exposures were associated with poorer chair-rise performance in both men and women, however, only associations between lifting and standing/walking and chair-rise remained statistically significant among men in the final model. Spline regression analyses showed non-linear associations and confirmed the findings.

Conclusions: Higher physical exposure throughout working life is associated with slightly poorer chair-rise performance. The associations between exposure and outcome were non-linear.

\section{INTRODUCTION}

In the 1980s, hard physical labour was thought to strengthen workers, but later studies showed that hard physical work could not maintain physical function in middle-aged workers. ${ }^{1}$ Since then, a history of hard physical work has been associated

\section{Strengths and limitations of this study}

Few studies evaluate the influence of exposures in working life on the musculoskeletal ageing process; therefore, this study contributes to new knowledge in the field.

- In this study, we have access to data from a large population-based cohort of middle-aged Danes.

- The strength of this study is the exposure assessment that sums all physical exposures during working life.

- One limitation of this study is the cross-sectional measure of physical function.

- Another limitation is the 'healthy worker' effect, which might be a bias in the study.

with lower physical function. In retrospective studies, old men with a history of manual work had lower physical performance ${ }^{2}$ and higher risk of physical disability ${ }^{3}$ compared to former non-manual workers; and, among retired miners, work strain was associated with impaired physical function. ${ }^{4}$ After 28 years follow-up, Leino-Arjas et a $\bar{l}$ found increased risk of poor physical function among those reporting high occupational physical strain at baseline and, in a retrospective study, Torgén et at found that a history of hard physical work could be both beneficial and detrimental to physical function.

The inconsistency of results, suggesting both beneficial and detrimental effects of physical exposures on midlife physical function, could be due to differences in study designs. Few follow-up studies have been conducted in this field, but despite the lack of long follow-up studies, there are signs of a 
negative association between physical exposures throughout working life and midlife physical function. Underlying biological processes could be the physiological explanation for this negative association, where acute changes in the musculoskeletal system might become chronic because of insufficient recovery time. ${ }^{78}$ In life course epidemiology, ${ }^{9}$ theories of cumulative exposures throughout life could be applied to occupational epidemiology, addressing physical wear and tear throughout working life as a possible risk factor in the musculoskeletal ageing process.

The inconsistency of research results in this field could also be due to bias in the exposure assessment. In previous studies in this field, interviews, ${ }^{2}$ combined with questionnaires, ${ }^{3}{ }^{6}$ information from registers ${ }^{3}$ and assessment by experts, ${ }^{4}$ have been used to categorise physical job strain, but few studies have included duration of exposure. In this study, we introduce a cumulative and continuous exposure assessment based on information from a job exposure matrix (JEM). To the best of our knowledge, this is the first study to use lifetime exposures in an analysis of midlife physical function assessing the influence of work on the musculoskeletal ageing process.

Inconsistency of research results in this field could also be due to the use of surrogate measures and the use of a variety of outcome measures. The musculoskeletal ageing process can be studied at different levels, from impairment in specific body systems (eg, muscle strength), through functional limitations, to disability. ${ }^{10}$ A test of chair-rise performance is independent of the surrounding environment and has been used worldwide to assess functional limitations in different age groups and settings, and performance tests are important predictors of morbidity ${ }^{11}$ and mortality in older people. ${ }^{12}$ However, chair-rise performance is seldom used in the occupational field as a dynamic measure.

Our study combines occupational epidemiology and exposure assessment with a well-known gerontological outcome measure in a large population-based cohort. This study was planned to include multiple outcome measures, but a literature review showed differences in associations between exposure and outcome measures: primarily a positive association between hard physical work and upper limb strength, ${ }^{13}$ and a negative association between hard physical work and lower limb strength/dynamic function. ${ }^{2-6}$ The underlying mechanisms could vary between the upper and the lower limb/ dynamic function, and, therefore, the outcome measures were separated in the analyses.

In the first paper, we found that physical exposures in working life had a minor but positive association with hand grip strength in middle-aged men. ${ }^{13}$

The aim of this study is therefore to evaluate the influence of physical exposures in working life on a dynamic measure of physical function. According to previous studies in this field, a history of hard physical work was hypothesised to be associated with lower physical performance in midlife and, thereby, with poorer performance in a chair-rise test.

\section{METHODS}

This population-based retrospective study included a cross-sectional physical examination as part of the Copenhagen Aging and Midlife Biobank (CAMB). ${ }^{14}$ CAMB was established in 2009-2011 to study signs of early ageing in middle-aged Danes, and was based on three existing Danish cohorts. In this study, we used data from two of the three cohorts in CAMB: 'The Metropolit Cohort' (MP) and 'The Danish Longitudinal Study on Work, Unemployment and Health' (DALWUH) group, in which 12656 middle-aged men and women were invited to participate (figure 1). ${ }^{15}$

The data collection in CAMB was made between April 2009 and March 2011, and included a postal questionnaire together with a health examination at the National Research Centre for the Working Environment (NRCWE). For details about the use of data from CAMB in this study and a description of the cohorts, see our research protocol. ${ }^{15}$ The selection and attrition in the study are illustrated in figure 1 .

\section{Exposure}

The assessment of physical exposures at work was based on information about job history from the questionnaire combined with data from a JEM. Self-reports of physical exposures in working life from the questionnaire were not used, since we found low reliability of that information in a previous study. ${ }^{16}$ The CAMB questionnaire provided job titles and length of service for participants' five longest held occupations. Each participant's job history was coded according to the 1988 revision of the Danish version of the International Standard Classification of Occupations register (D-ISCO 88) ${ }^{15}$ From a Danish JEM (the Lower Body JEM), information about physical exposures in Danish jobs (linked to D-ISCO 88 codes) was retrieved. ${ }^{17}$ The Lower Body JEM is based on expert judgments of physical exposures associated with risk of osteoarthritis in the lower limbs: sitting, standing/walking, whole body vibration, kneeling and lifting (weight and number of heavy lifts). ${ }^{17}$ In the present study, we used information about three physical exposures: (1) lifting; the main physical exposure included in the definition of hard physical work, ${ }^{18}$ (2) standing/walking; a common exposure in jobs categorised as physically demanding, but without lifting (eg, cleaning assistants) and (3) kneeling, because kneeling at work places demands on muscle power and strength in the lower limbs.

The total amount of exposure for a study participant was expressed as the number of years incurred by a standard daily exposure. Thus, the years of employment in each of the jobs retrieved from the questionnaire were multiplied by the corresponding daily amount of lifting, standing/walking and kneeling retrieved from the Lower Body JEM, and then calculated for the 
Three existing Danish Cohorts in Copenhagen Aging and Midlife Biobank (CAMB)

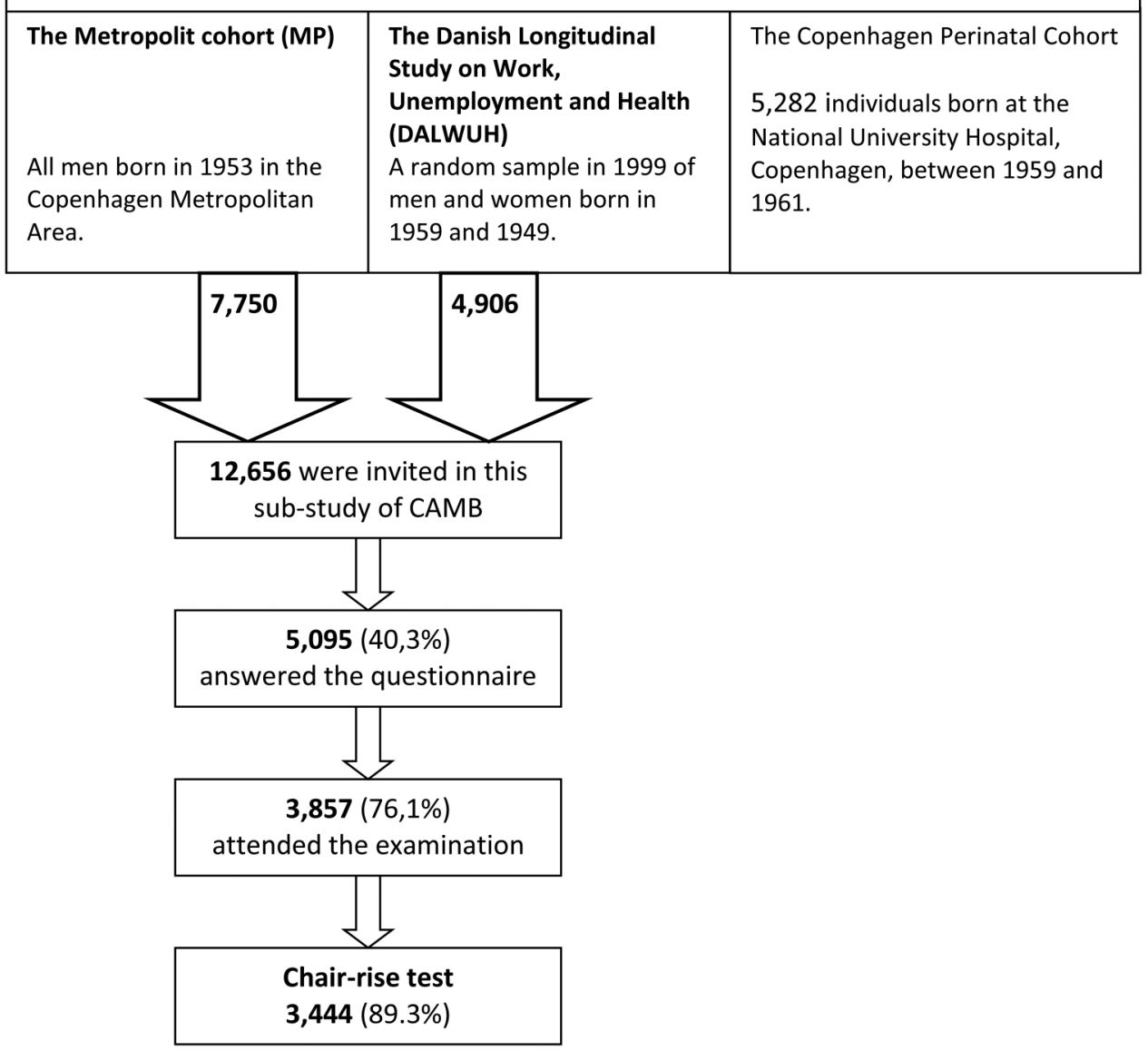

Figure 1 Copenhagen Aging and Midlife Biobank. Cohorts and participation.

participants' entire working life. In this way, exposures were standardised as ton-years (lifting $1000 \mathrm{~kg}$ each day in 1 year), stand-years (standing/walking at work for $6 \mathrm{~h}$ each day in 1 year) and kneel-years (kneeling at work for $1 \mathrm{~h}$ each day in 1 year).

\section{Outcome}

Chair-rise performance was measured as the number of chair-rises performed during a $30 \mathrm{~s}$ test. The test was performed using a chair (height $45 \mathrm{~cm}$ ) with a mechanical contact in the seat, enabling automatic recording of the number of posture transitions and the number of cycles completed, for example, 21.2 cycles in $30 \mathrm{~s}^{19}$ As the test was somewhat tiresome each participant made only a single attempt.

\section{Covariates}

From the CAMB questionnaire, information about vocational education was categorised into five groups: unskilled, skilled manual worker and short, medium, or long cycles further education. Men were included from two cohorts (MP and DALWUH), and since the two cohorts differed according to scope and social background, the variable 'cohort' was included as a confounder. The questionnaire provided information about the number of chronic diseases among participants, and these were grouped into three: 0,1 and $\geq 2$ or more chronic diseases. The diseases considered relevant were asthma, diabetes, hypertension, angina pectoris, stroke, myocardial infarction, bronchitis, emphysema, rheumatoid arthritis, osteoarthritis, cancer, anxiety, depression/ other psychiatric diseases and back pain. Leisure-time physical activity (LTPA) was categorised as medium/ hard: $>4 \mathrm{~h}$ a week; light: $<4 \mathrm{~h}$ a week and sedentary activity: reading/watching television. Smoking history was calculated as pack-years (defined as 20 cigarettes or an equal amount of tobacco smoked each day for 1 year), and current alcohol consumption was categorised in units of alcohol per week. Pain in nine regions of the body was summarised (neck, shoulders, upper back, elbows, lumbar region, hands/wrists, hips, knees and ankles); the minimum score was 9 (no pain in any of the regions) and the maximum was 81 (worst possible pain in all 9 regions). Work status was defined as employed or unemployed (currently unemployed and early or disability retirement).

\section{Theoretical model}

A theoretical model was established based on the hypothesis of physical exposures during working life 
influencing chair-rise performance in combination with the effect of 'wear and tear' and ageing. Age, height, cohort and vocational education were seen as confounders in the theoretical model (figure 2). Chronic diseases influence physical function but could be both a confounder and a mediator since a chronic disease could be caused by the exposure, or morbidity could influence the duration and intensity of exposure in working life. LTPA is beneficial to physical function in general, but the association with work exposures is less clear. Current LTPA could be a mediator in the association between physical exposures at work and physical function. However, current LTPA, as a proxy of former LTPA, could also influence how many years a worker is able to meet the demands of a hard physical job and, thereby, influence the total amount of exposure. Alcohol and smoking were seen as mediators in the conceptual model together with pain and work status.

\section{Statistical analysis}

As the effects of physical exposures were assumed to be gender-specific, all analyses were performed separately for each sex, as suggested by Silverstein et al. ${ }^{20}$ Both unadjusted and adjusted associations between exposures (summation of exposure-years) and outcome (number of chair-rises) were assessed in general linear regression models. Unadjusted analyses were assessed and, afterwards, age, height, cohort and vocational education were included (model 1). Subsequently, chronic disease and LTPA were included in a second series of multivariable models to study their mediation effect. Finally, all mediators were included in a third series of multivariable models to study if an observed effect could be explained by the mediators. All analyses were performed in PROC GLM (SAS V.9.2). In order to study how well the models predicted physical performance, we reported the proportion of the variation explained by the regression models $\left(\mathrm{R}^{2}\right){ }^{21}$
Since the effect of physical exposure on physical performance has been suggested to be both beneficial and detrimental to physical function, a linear term may be too limited to characterise these associations. ${ }^{22}$ Exposure was therefore categorised in quartiles, and analyses were repeated. On the basis of those results, a statistician suggested studying the shape of the associations by modelling them as restricted cubic spline functions in model 1. The resulting spline functions were then plotted to show the expected difference in outcome attributed to each category of exposure, avoiding a linearity assumption. $^{22} 23$

Attrition analyses were performed in the CAMB cohort $^{14}$ and, in this study, differences in exposure characteristics between participants and non-participants were analysed with t tests.

All analyses were performed using SAS V.9.2, except the regression with spline functions, which was performed in the $\mathrm{R}$ system for statistical computation (http://www.cran.r-project.org).

The power calculation is found in online supplementary information.

\section{RESULTS}

The characteristics of the study population are presented in table 1 . Mean age was 59 years for men as well as for women, and since the MP cohort included only male participants, men constitute $79.2 \%$ of the study population. Women were exposed to fewer exposureyears compared to men, particularly with regard to kneel-years. Mean seniority at work based on the five longest held phases of employment was almost similar between the two sexes: $31.46(\mathrm{SD}=8.12)$ years in men and $29.69(\mathrm{SD}=8.94)$ years in women, although fewer women were still in the labour market $(77.0 \%$ vs $88.0 \%)$. Women achieved, on average, 1.2 chair-rises less than men in the $30 \mathrm{~s}$ test $(20.38(\mathrm{SD}=5.33)$ versus 21.58 $(\mathrm{SD}=5.60)$ ), but $94.4 \%$ of the women completed the test

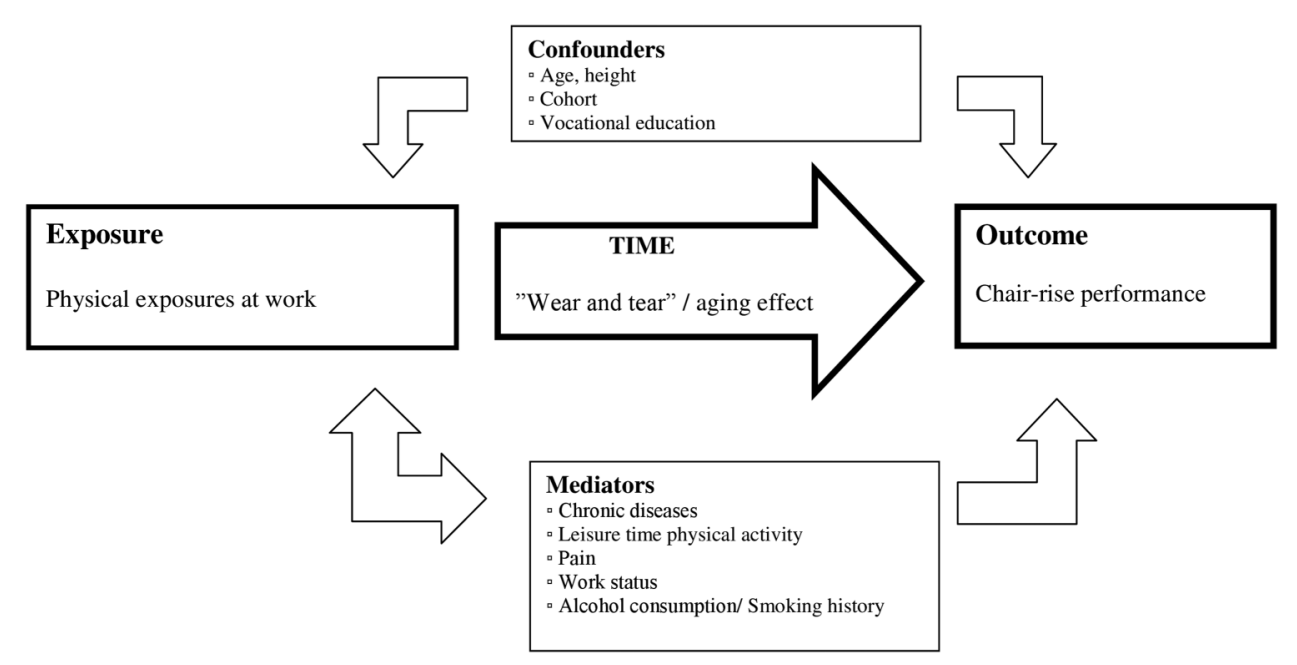

Figure 2 Theoretical model. Associations between exposure and outcome, including covariates. Gender is not included in the model since each gender is analysed separately. 
Table 1 Characteristics of the study population, exposures and outcome

\begin{tabular}{|c|c|c|c|c|c|c|}
\hline & \multicolumn{3}{|l|}{ Men } & \multicolumn{3}{|l|}{ Women } \\
\hline & $\mathbf{N}(\%)$ & Median (IQR) & Mean (SD) & $\mathbf{N}(\%)$ & Median (IQR) & Mean (SD) \\
\hline Age & 4035 & $59(59-59)$ & $59.0(2.3)$ & 1060 & $63(53-63)$ & $58.6(5.0)$ \\
\hline Height, cm & 3968 & $180(175-184)$ & $179.7(6.8)$ & 1045 & $167(163-170)$ & $166.6(6.2)$ \\
\hline Smoking, pack-years & 3842 & $12.5(0-33)$ & $20.7(26.7)$ & 999 & $2(0-18)$ & $10.5(17.5)$ \\
\hline Alcohol consumption, Units/Week & 3973 & $12(6-20)$ & $14.8(14.0)$ & 1033 & $6(2-10)$ & $8.0(12.1)$ \\
\hline Pain index ${ }^{*}$ & 3990 & $17(12-24)$ & 19.9 (10.9) & 1053 & $20(14-30)$ & $23.6(13.1)$ \\
\hline Chronic diseasest & 3993 & & & 1052 & & \\
\hline No disease & $1225(30.7)$ & & & $320(30.4)$ & & \\
\hline 1 disease & 1326 (33.2) & & & $311(29.6)$ & & \\
\hline 2 or more diseases & $1442(36.1)$ & & & $421(40.0)$ & & \\
\hline Vocational education & 3964 & & & 1039 & & \\
\hline Long cycle & 738 (18.6) & & & 131 (12.6) & & \\
\hline Medium cycle & $857(21.6)$ & & & $313(30.1)$ & & \\
\hline Short cycle & $336(8.5)$ & & & 107 (10.3) & & \\
\hline Semiskilled & $1689(42.6)$ & & & 387 (37.2) & & \\
\hline Unskilled & $344(8.7)$ & & & $101(9.7)$ & & \\
\hline Leisure-time physical activity $\ddagger$ & 3957 & & & 1040 & & \\
\hline Medium/hard & 1253 (31.7) & & & $255(24.5)$ & & \\
\hline Light & $2240(56.6)$ & & & 706 (67.9) & & \\
\hline Sedentary & 464 (11.7) & & & $79(7.6)$ & & \\
\hline Labour market status & 3953 & & & 1033 & & \\
\hline Employed & 3479 (88.0) & & & $802(77.6)$ & & \\
\hline Unemployed§ & 474 (12.0) & & & $231(22.4)$ & & \\
\hline Cohort & 4035 & & & 1060 & & \\
\hline $\mathrm{MP}$ & $3153(78.1)$ & & & & & \\
\hline DALWUH & $882(21.9)$ & & & $1060(100.0)$ & & \\
\hline Ton-years** & 3880 & $2.3(0-16)$ & $12.9(23.1)$ & 1016 & $0.0(0-7.9)$ & $6.0(12.4)$ \\
\hline Stand-years $† \dagger$ & 3880 & $3.9(0-22)$ & $11.3(13.8)$ & 1016 & $0.0(0-13)$ & 7.4 (11.4) \\
\hline Kneel-yearsł & 3880 & $0.0(0-0)$ & 7.3 (15.0) & 1016 & $0.0(0-0)$ & $1.1(3.0)$ \\
\hline \multicolumn{7}{|c|}{$\begin{array}{l}\text { *Summation of pain in nine regions of the body. Minimum score is } 9 \text { (no pain in any of the regions) and maxi } \\
\text { all } 9 \text { regions). } \\
\text { †Asthma, diabetes, hypertension, angina, stroke, bronchitis, chronic obstructive pulmonary disease, rheumat } \\
\text { anxiety, depression, psychiatric diseases and back disease. } \\
\ddagger \text { Medium/hard : }>4 \mathrm{~h} \text { a week; light: }<4 \mathrm{~h} \text { a week; sedentary: reading/watching television in leisure time. } \\
\text { §Unemployed=currently unemployed and early retirement, disability pensioners, etc. } \\
\text { ๆMale participants were from two cohorts. } \\
\text { **Amount of lifting during working life. One ton-year is lifting } 1000 \mathrm{~kg} \text { each day for } 1 \text { year. } \\
\dagger † T o t a l \text { exposure to standing/walking at work. One stand-year is standing/walking for } 6 \mathrm{~h} \text { each day in } 1 \text { year. } \\
\text { †¥Total exposure to kneeling at work. One kneel-year is kneeling at work for } 1 \mathrm{~h} \text { each day in } 1 \text { year. } \\
\text { DALWUH, Danish Longitudinal Study on Work, Unemployment and Health; MP, Metropolit Cohort. }\end{array}$} \\
\hline
\end{tabular}

compared to $88.4 \%$ of men. At the physical examination, whether or not participants had a specific reason for not performing in the physical test was noted. The most common reasons were recent surgery and disability in general.

In general, there was a negative association between exposure-years and chair-rise in men and in women. Exposure to ton-years, stand-years and kneel-years was associated with poorer chair-rise performance in unadjusted analyses. Introducing age, height, cohort and vocational education attenuated the effect of tonyears and kneel-years in men, whereas the effect of physical exposures was slightly increased in women (table 2).

Introducing chronic diseases in model 1 attenuated the effect of exposure on chair-rise performance in men and in women. Introducing LTPA in model 1 did not change the associations in men, and did so only to a small extent in women. Inclusion of all covariates in the final model attenuated the associations, and only the associations between ton-years/stand-years and chair-rise in men remained statistically significant.

Spline regression analyses visualising model 1 confirmed the findings from the linear analyses, and a negative association between exposure to ton-years/ stand-years and chair-rise was observed in men (figure $3)$. The effect reached a maximum decrease of -1.83 (95\% CI: $(-2.70$ to -0.95$)$ ) chair-rises in men exposed to 30 ton-years, compared to men without this occupational exposure. This association was non-linear, and further exposure to ton-years did not decrease chair-rise performance.

In women, associations between exposure-years and chair-rise were non-linear and had broad CIs, due to few participants with higher exposures (figure 3). An analysis of the association between exposure to ton-years 
Table 2 Multivariable linear regression models

\begin{tabular}{|c|c|c|c|c|c|c|c|}
\hline \multirow[b]{3}{*}{ Exposure } & \multirow[b]{3}{*}{ Model } & \multicolumn{3}{|l|}{ Men* } & \multicolumn{3}{|c|}{ Woment } \\
\hline & & \multicolumn{3}{|c|}{ Chair-rise number in $30 \mathrm{~s}$} & \multicolumn{3}{|c|}{ Chair-rise number in $30 \mathrm{~s}$} \\
\hline & & $\begin{array}{l}\text { Regression } \\
\text { coefficient }\end{array}$ & p Value & $\mathbf{R}^{2} \ddagger(\%)$ & $\begin{array}{l}\text { Regression } \\
\text { coefficient }\end{array}$ & p Value & $R^{2} \ddagger(\%)$ \\
\hline \multirow[t]{5}{*}{ Ton-years§ } & Unadjusted & -0.0401 & $<0.0001$ & 2.4 & -0.0364 & 0.0426 & 0.6 \\
\hline & Model 1ๆ & -0.0382 & $<0.0001$ & 10.24 & -0.0454 & 0.0068 & 14.99 \\
\hline & Model 1 and chronic diseases ${ }^{\star \star}$ & -0.0328 & $<0.0001$ & 11.83 & -0.0363 & 0.0271 & 18.81 \\
\hline & $\begin{array}{l}\text { Model } 1 \text { and leisure-time physical } \\
\text { activity } \dagger \dagger\end{array}$ & -0.0388 & $<0.0001$ & 13.77 & -0.419 & 0.0113 & 18.0 \\
\hline & 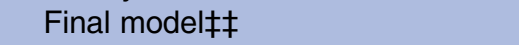 & -0.0156 & 0.0030 & 19.1 & -0.0198 & 0.2485 & 24.1 \\
\hline \multirow[t]{5}{*}{ Stand-years§§ } & Unadjusted & -0.0596 & $<0.0001$ & 2.08 & -0.0432 & 0.0271 & 0.7 \\
\hline & Model 1ๆ & -0.0610 & $<0.0001$ & 10.2 & -0.0470 & 0.0100 & 14.9 \\
\hline & Model 1 and chronic diseases ${ }^{\star *}$ & -0.0523 & $<0.0001$ & 11.8 & -0.0393 & 0.0278 & 18.8 \\
\hline & $\begin{array}{l}\text { Model } 1 \text { and leisure-time physical } \\
\text { activity }+\dagger\end{array}$ & -0.0610 & $<0.0001$ & 13.66 & -0.0474 & 0.0083 & 18.1 \\
\hline & Final modelł‡ & -0.0207 & 0.0222 & 19.0 & -0.0262 & 0.1548 & 24.2 \\
\hline \multirow[t]{5}{*}{ Kneel-yearsๆી } & Unadjusted & -0.0365 & $<0.0001$ & 0.87 & -0.1010 & 0.1618 & 0.29 \\
\hline & Model 1ๆ & -0.0348 & $<0.0001$ & 8.89 & -0.1477 & 0.0286 & 14.67 \\
\hline & Model 1 and chronic diseases ${ }^{* *}$ & -0.0268 & 0.0004 & 10.75 & -0.1408 & 0.0326 & 18.77 \\
\hline & $\begin{array}{l}\text { Model } 1 \text { and leisure-time physical } \\
\text { activity }+\dagger\end{array}$ & -0.0366 & $<0.0001$ & 12.43 & -0.1421 & 0.0322 & 17.77 \\
\hline & Final modelł‡ & -0.0053 & 0.4961 & 18.9 & -0.0708 & 0.2858 & 24.1 \\
\hline 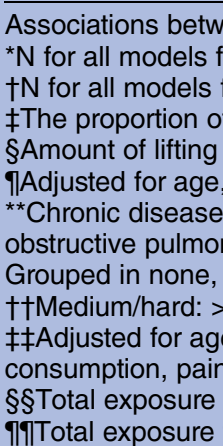 & $\begin{array}{l}\text { een exposure-years and chair-rise perform } \\
\text { or men is } 2463 \text {. } \\
\text { or women is } 666 \text {. } \\
\text { the variation explained by the regression r } \\
\text { in working life. One ton-year is lifting } 1000 \\
\text { height, cohort and vocational education. } \\
\text { s in three groups: } 0,1 \text { or } \geq 2 \text { of the followin } \\
\text { lary disease, rheumatoid arthritis, osteoarth } \\
\text { one, two or more chronic diseases. } \\
4 \mathrm{~h} \text { a week; light: }<4 \mathrm{~h} \text { a week; sedentary: } \\
\text {, height, cohort, vocational education, chro } \\
\text { index. } \\
\text { to standing/walking at work. One stand-yea }\end{array}$ & $\begin{array}{l}\text { nce. } \\
\text { odel in \%. } \\
\text { g each day for } 1 \\
\text { diseases: asthm } \\
\text { itis, cancer, anx } \\
\text { ading/watching } \\
\text { ic diseases, leis } \\
\text { is standing/walk }\end{array}$ & $\begin{array}{l}\text { year. } \\
\text { a, diabetes, } \\
\text { ty, depress } \\
\text { levision in } \\
\text { ung for } 6 \mathrm{~h} \text { e }\end{array}$ & $\begin{array}{l}\text { nypertens } \\
\text { on, psych } \\
\text { isure tim } \\
\text { ical activi } \\
\text { ch day in }\end{array}$ & $\begin{array}{l}\text {, angina, stroke } \\
\text { ric diseases an } \\
\text { smoking histor } \\
\text { year. }\end{array}$ & $\begin{array}{l}\text { bronchitis } \\
\text { back dise } \\
\text { alcohol }\end{array}$ & hronic \\
\hline
\end{tabular}

and chair-rise indicates a negative association with a maximum in women exposed to 20 ton-years $(-2.45$ chair-rises CI (-3.92 to -0.98$))$.

\section{DISCUSSION}

We hypothesised that physical exposures throughout working life were associated with impaired physical function in midlife, measured by chair-rise performance, and that the theoretical explanation could be wear and tear or accumulation of exposures during life. We found that the influence of physical exposures on physical function was relatively small and varied between genders.

In general, our results are in line with previous findings of poorer physical performance among men with a history of hard physical work, ${ }^{2-6}$ but our cohort included younger participants compared to the studies by Russo et $a l^{2}$ and Cassou et $a l^{3}$ Our study design is comparable to the retrospective cohort design of Torgén et $a l^{6}{ }^{6}$ who found that long-lasting physical demands were associated with poorer dynamic muscle function, though our study is larger. Chair-rise performance, as a measure of dynamic function, relies on muscle power in the lower limbs, which is known to decrease due to musculoskeletal ageing. ${ }^{24}$ The observed decrease in chair-rise performance in exposed men could hypothetically be a sign of an accelerated musculoskeletal ageing process, caused by exposures at work. However, the proportion of the variation explained by the models was low, and the proportion of the variance in outcome measures explained by the physical exposures was also low. In linear models (model 1), a loss of 0.04 chair-rise/ton-year was seen in men, which equals a loss of 1.2 chair-rises in 30 years and a loss of 0.045 chair-rise/ton-year in women, which again equals a loss of 0.9 chair-rise in 20 years. The non-linear analyses showed higher effects: a loss of 1.8 chair-rises among those exposed to 30 ton-years compared to unexposed and 2.5 chair-rises less among women exposed to 20 ton-years. In the final model, the results were attenuated, especially among women. The question is, whether these findings are clinically relevant, and follow-up studies in this cohort should examine the influence of physical performance on work ability, sickness absence, early retirement and death. A recent 13-year follow-up study in the British birth cohort showed a higher 

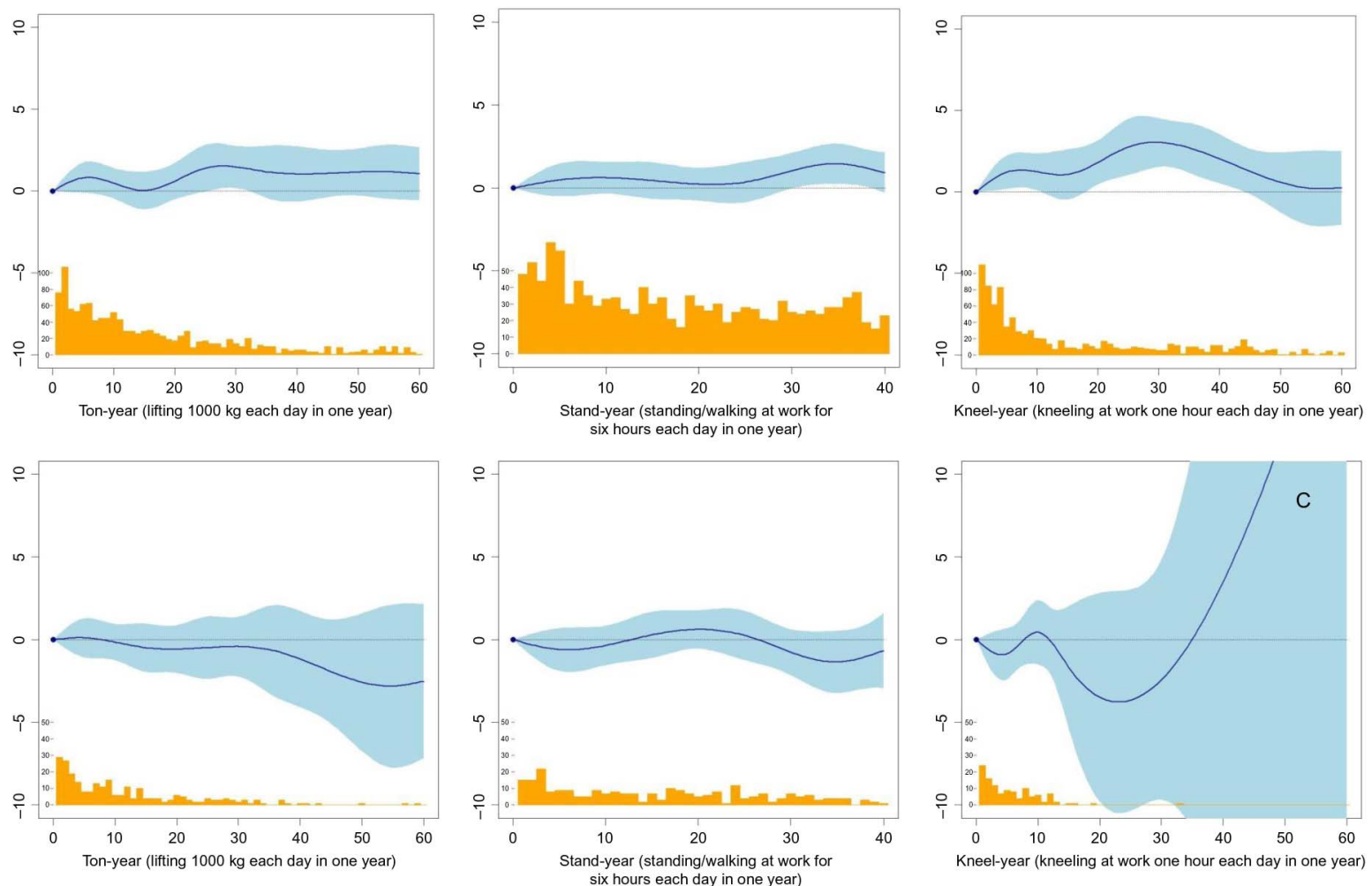

Figure 3 Multivariable non-linear (spline) regressions including 95\% Cls. Associations between exposure-years and number of chair-rises/30 s. Model 1 including age, height, cohort and vocational education. Upper row: men. Lower row: women. The number of participants is along the $\mathrm{x}$-axis and indicated by orange bars.

mortality rate among those with the lowest physical capability at 53 years of age. ${ }^{25}$

Covariates were introduced to the models separately, and whether covariates were confounders or mediators was discussed. However, the overall conclusion in this study is that inclusion of all covariates in the linear regression models attenuated the associations. Chronic diseases and differences in physical activity in leisure time marginally explained the differences in performance.

In general, performance in the chair-rise test was lower in women and men with physical exposures in working life. In contrast to our findings, other studies in this field found that negative associations between work load and physical performance were more pronounced in women than in men. ${ }^{6}$ We have no obvious explanation for the observed differences in associations between men and women in this cohort, relating to their performance in the chair-rise test. Little is known about the gender difference in the influence of work on the musculoskeletal ageing process. The job exposure matrix was not gender-specific, as has been suggested by Solovieva et $a l,{ }^{26}$ and this could introduce misclassification bias due to differences in exposure between men and women with the same job-titles. However, this cohort included few women with a history of hard physical work (see the broad CIs in figure 3) and this could be part of the explanation of our results.

\section{Strengths and limitations}

The exposure assessment was a strength of this study, compared to other retrospective studies of lifetime physical workload, because the assessment included both intensity and duration of exposure. If a linear association was found, a threshold for exposure years could be calculated. However, our results indicate that the variation in physical function is caused by multiple factors, and exposures at work may play a minor role. Furthermore, the associations turned out to be non-linear. Standardisation of exposure to lifting could introduce measurement bias, since 20 ton-years can be 'earned' in only 10 years of heavy work, or 40 years of less heavy work and intensity. According to the exposure assessment, the use of a job exposure matrix was thought to improve the validity of exposure assessment, but it has not been studied. The primary aim of the Lower Body JEM was to study osteoarthritis in the lower limbs, and it focused on exposures from lifting, kneeling, vibration and standing/walking at work. The aim of this study was to study physical function, and we have claimed that, especially, lifting and standing/walking at work could influence musculoskeletal ageing and chair-rise performance. However, this, as well, has not been studied by others.

Another possible bias in this study is the risk of misclassification of exposure in job exposure matrices, where exposures are assigned to exposure groups 
thought to be homogeneous. Objective measures in bigger cohorts are preferable in future epidemiological studies in this field. ${ }^{27}$

The large study population was a strength, although the low response-rate in the CAMB study could have introduced bias due to selective drop-out. The attrition analyses showed that participants had lower exposures compared to non-participants. ${ }^{13}$ Furthermore, sub-group analyses showed that participants not attending the chair-rise test had a lower socioeconomic status and more exposure-years compared to the participants fulfilling this test. This could attenuate the results further. In a future study, we will examine the associations between physical exposures in working life and self-reports of mobility among respondents to the CAMB questionnaire. In this way, we will be able to compare mobility among participants and non-participants through a selfreported measure of physical function.

Another possible bias is the 'healthy worker effect', where those participants having 'earned' the longest or highest exposures throughout working life could be a special sub-group of workers. ${ }^{28}$ This effect is perhaps seen in the non-linear associations as a less pronounced detrimental effect of maximum exposure.

\section{Conclusion}

In this cohort, greater duration of physical exposures throughout adult life was associated with poorer chairrise performance in men with a mean age of 59 years. In women, exposures were associated with poorer chairrise performance in unadjusted analyses, but few female participants had physical exposures in working life. The influence of physical exposure on midlife physical function was numerically small and non-linear.

\section{Author affiliations}

${ }^{1}$ Department of Occupational Medicine, Copenhagen University Hospital Holbæk, Holbæk, Denmark

${ }^{2}$ National Research Centre for the Working Environment, Copenhagen, Denmark

${ }^{3}$ Department of Public Health, The Research Unit for General Practice and Section of General Practice, University of Copenhagen, Copenhagen, Denmark ${ }^{4}$ Department of Public Health, University of Copenhagen, Section of Social Medicine, Copenhagen, Denmark

${ }^{5}$ Center for Healthy Aging, University of Copenhagen, Copenhagen, Denmark ${ }^{6}$ Department of Occupational Medicine, Danish Ramazzini Center, Regional Hospital West Jutland-University Clinic, Herning, Denmark

Acknowledgements The authors would like to thank Helle Bruunsgaard, Nils-Erik Fiehn, Poul Holm-Pedersen, Erik Lykke Mortensen and Merete Osler, who initiated and established the Copenhagen Aging and Midlife Biobank from 2009-2011 together with Kirsten Avlund, Åse Marie Hansen and Rikke Lund. They also acknowledge the crucial role of the initiators and steering groups of the Metropolit Cohort, The Copenhagen Perinatal Cohort and The Danish Longitudinal Study on Work Unemployment and Health. They also acknowledge the contributions of Willy Karlslund and Drude Molbo (data handling/processing). They also acknowledge Tine Steen Rubak and her supervisors for their important contribution in the establishment of the Lower Body JEM.

Contributors AM planned the project together with SR, OSM, JHA and KA. AM wrote the draft of the article, and all the authors contributed by commenting on the manuscript. VS assisted in the power calculation and performed the spline regression analyses. LLA and AMH were responsible for designing and implementing the physical tests in CAMB. KA was the principal investigator $(\mathrm{PI})$ and $\mathrm{RL}$ was the main $\mathrm{Co}-\mathrm{PI}$ of the CAMB study, and, after the death of $K A, R L$ is the PI of CAMB. AM revised the article together with VS. All the authors approved the final manuscript.

Funding This study was supported by a grant from The Research Foundation for the Working Environment, Denmark. The foundation has no influence on the data handling or manuscript approval. The Copenhagen Aging and Midlife Biobank has been supported by a generous grant from the VELUX FOUNDATION.

\section{Competing interests None declared.}

Ethics approval Videnskabsetisk Komite Frederiksberg og KÃ, Koebenhavn Kommune.

Provenance and peer review Not commissioned; externally peer reviewed.

Data sharing statement No additional data are available.

Open Access This is an Open Access article distributed in accordance with the Creative Commons Attribution Non Commercial (CC BY-NC 4.0) license, which permits others to distribute, remix, adapt, build upon this work noncommercially, and license their derivative works on different terms, provided the original work is properly cited and the use is non-commercial. See: http:// creativecommons.org/licenses/by-nc/4.0/

\section{REFERENCES}

1. Nygård C, Luopajärvi T, Ilmarinen J. Musculoskeletal capacity and its changes among aging municipal employees in different work categories. Scand J Work Environ Health 1991;17:110-17.

2. Russo A, Onder G, Cesari M, et al. Lifetime occupation and physica function: a prospective cohort study on persons aged 80 years and older living in a community. Occup Environ Med 2006;63:438-42.

3. Cassou B, Derriennic F, Iwatsubo Y, et al. Physical diability after retirement and occupational risk factors during working life: a cross sectional epidemiological study in the Paris area. J Epidemiol Community Health 1992;46:506-11.

4. Calmels P, Ecochard R, Blanchon MA, et al. Relation between locomotion impairment, functional independence in retirement, and occupational strain resulting from work carried out during working life. Study of a sample population of 350 miners in the Loire valley in France. J Epidemiol Community Health 1998;52:283-8.

5. Leino-Arjas $\mathrm{P}$, Solovieva $\mathrm{S}$, Riihimäki $\mathrm{H}$, et al. Leisure time physical activity and strenuousness of work as predictors of physical functioning: a 28 year follow up of a cohort of industrial employees. Occup Environ Med 2004;61:1032-8.

6. Torgén M, Punnett L, Alfredsson L, et al. Physical capacity in relation to present and past physical load at work: a study of 484 men and women aged 41 to 58 years. Am J Ind Med 1999;36:388-400.

7. Mohren DC, Jansen NW, Kant I. Need for recovery from work in relation to age: a prospective cohort study. Int Arch Occup Environ Health 2010;83:553-61.

8. De Croon EM, Sluiter JK, Frings-Dresen MH. Need for recovery after work predicts sickness absence: a 2-year prospective cohort study in truck drivers. J Psychosom Res 2003;55:331-9.

9. Kuh D, Ben-Shlomo Y. A life course approach to chronic diseases epidemiology. Oxford Medical Publishers, 2004.

10. Verbrugge LM, Jette AM. The disablement process. Soc Sci Med 1994;38:1-14.

11. Cooper R, Kuh D, Cooper C, et al. Objective measures of physical capability and subsequent health: a systematic review. Age ageing 2011;40:14-23.

12. Cooper R, Kuh D, Hardy R. Objectively measured physical capability levels and mortality: systematic review and meta-analysis. BMJ 2010;341:c4467.

13. Møller A, Reventlow S, Hansen Å, et al. Does a history of physical exposures at work affect hand-grip strength in strength in midlife? A retrospective cohort study in Denmark. Scand J Work Environ Health 2013;39:599-608.

14. Avlund K, Osler M, Mortensen EL, et al. Copenhagen Aging and Midlife Biobank (CAMB). An introduction. J Aging Health 2014;26:5-20.

15. Møller A, Mortensen OS, Reventlow S, et al. Lifetime occupational physical activity and musculoskeletal aging in middle-aged men and 
women in Denmark: retrospective cohort study protocol and methods. JMIR Res Protoc 2012;1:e7.

16. Møller A, Reventlow S, Andersen JH, et al. Validity of workers' self-reports. Evaluation of a question assessing lifetime exposure to occupational physical activity. Br J Med Med Res 2012;2:536-52.

17. Rubak T, Svendsen S, Andersen J, et al. An expert-based job exposure matrix for large scale epidemiologic studies of primary hip and knee osteoarthritis: the Lower Body JEM. BMC Musculoskelet Disord 2014; 15:204.

18. Mateson $\mathrm{L}$. The functional capacity evaluation. In: Anderson G Demeter S, Smith G, eds. Chicago: Disability Evaluation. Mosby Year, 2003.

19. Hansen Å, Andersen L, Skotte J, et al. Social class differences in physical functions in middle-aged men and women. $J$ Aging Health 2014:26:88-105.

20. Silverstein B, Fan Z, Smith C, et al. Gender adjustment or stratification in discerning upper extremity musculoskeletal disorder risk? Scand J Work Environ Health 2009;35:113-26.

21. Altman DG. Practical statistics for medical research. London: Chapman \& Hall, 1999.
22. Greenland S. Dose-response and trend analysis in epidemiology: alternatives to categorical analysis. Epidemiology 1995;6:356-65.

23. Steenland K, Deddens J. A practical guide to dose-response analyses and risk assessment in occupational epidemiology. Epidemiology 2004;15:63-70.

24. Reid KF, Fielding RA. Skeletal muscle power: a critical determinant of physical functioning in older adults. Exerc Sport Sci Rev 2012;40:4-12.

25. Cooper R, Strand B, Hardy R, et al. Physical capability in mid-life and survival over 13 years of follow-up: British birth cohort study. BMJ 2014;348:g2219.

26. Solovieva S, Pehkonen I, Kausto J, et al. Development and validation of a job exposure matrix for physical risk factors in low back pain. PLOS ONE 2012;7:e48680.

27. Skotte J, Korshøj M, Kristiansen J, et al. Detection of physical activity types using triaxial accelerometers. J Phys Act Health 2014; 11:76-84.

28. Kenny G, Yardley J, Martineau L, et al. Physical work capacity in older adults: implications for the aging worker. Am J Ind Med 2008:51:610-25. 